\title{
A promoção do Consumo Sustentável por um movimento de Transição
}

\author{
Minelle Enéas da Silva \\ Universidade Federal do Rio Grande do Sul - minele.adm@gmail.com \\ Tânia Nunes Silva \\ Universidade Federal do Rio Grande do Sul - tania.silva@ufrgs.br
}

\begin{abstract}
RESUMO
Considerando a necessidade de um processo contínuo e sistêmico de mudança - 'transições' - em busca de um novo contexto rumo à sustentabilidade, o objetivo deste artigo é compreender como a transição para a sustentabilidade, em meio a um contexto de crise, pode auxiliar para a promoção do consumo sustentável. A partir de um ensaio teórico, busca-se aproximar duas abordagens que podem ser entendidas como complementares e emergentes direcionadas para uma nova dinâmica social. Para tanto, a discussão apresentada destaca que por meio de ações multi atores, a partir de uma perspectiva multi níveis, é possível a institucionalização de práticas responsáveis direcionadas a reconfigurações na sociedade. Com argumentos claros, o presente estudo apresenta contribuições para melhor entendimento da temática.
\end{abstract}

Palavras-chave: Consumo Sustentável; Transição Sócio-técnica; Inovação.

\section{Promoting Sustainable Consumption by a movement of Transition}

\begin{abstract}
Considering the need for a systemic and continuous process of change - 'transitions' - seeking of a new environment towards sustainability, the goal of this article is to understand how the transition to sustainability in the midst of a crisis context, can help to the promotion of sustainable consumption. From a theoretical essay, we seek to approximate two approaches that can be understood as complementary and emerging directed to a new social dynamic. Therefore, the discussion presented highlights that through actions multi actors, from a multi levels, it is possible the institutionalization of responsible practices aimed at reconfigurations in society. With clear arguments, this study presents contributions to better understanding of the subject.
\end{abstract}

Keywords: Sustainable Consumption; Socio-technical Transition; Innovation.

\section{Introdução}

As transformações que vêm sendo observadas em diferentes âmbitos no mundo, que são resultantes das práticas produtivas insustentáveis e do alto consumo da população, estão gerando grandes impactos sobre os recursos naturais. Em meio a esse contexto, cada vez mais as demandas por soluções profundas e efetivas estão se tornando salientes na sociedade, visto que as modificações incrementais e pontuais relacionadas aos problemas até então observados não conseguem contribuir para uma perspectiva mais clara de mudança. Isso porque segundo Geels (2010), os problemas que têm surgido com maior impacto do ser humano no meio, não conseguem mais ser solucionados com o desenvolvimento de tecnologias mais limpas. Pelo contrário, para que haja uma efetiva 'transição', respostas mais substantivas são necessárias. 
Vive-se, atualmente, imerso em uma crise no sistema de produção que, ao não conseguir vender o que é produzido e gerar lucros, demonstra-se incapaz de se sustentar ao longo do tempo (ONARAN, 2010; VANDEPITTE, 2011). Assim, questionamentos guiam as discussões correntes: Seria o momento de surgir nova forma de produção? Deve-se buscar manter o capitalismo? Nada concreto ainda se pode afirmar. O que se reconhece de forma clara é que o chamado desenvolvimento sustentável surge como alternativa viável à medida que cria novas relações rumo a um processo de transformação. A ideia para efetivar a sustentabilidade permeia o pensamento de buscar uma nova dinâmica social, na qual relações sociais são reconstruídas para uma visão mais coletiva (FOLADORI, 2005).

Sob esse campo de argumentação, no qual novas formas de se observar os contextos social e de mercado podem ser elaboradas, surge a discussão sobre a abordagem teórica de transição para a sustentabilidade, como uma área emergente que visa delinear quais seriam os melhores posicionamentos a serem assumidos, bem como qual seria o papel da inovação e de outros aspectos gerenciais para uma nova dinâmica na sociedade. Existem diferentes linhas de pensamento que permeiam essa vertente teórica. Com isso, assim como argumentam Lawhon e Murphy (2010), para a presente discussão na busca de um caminho de transição para um futuro mais sustentável, baseiase na perspectiva multi nível (PMN) que leva em consideração as influências diretas sobre a sociedade (GEELS, 2002; 2011; SMITH, VOß; GRIN, 2010).

Para melhor compreensão, tem-se que as transições para a sustentabilidade são de longo prazo, multi dimensionais e assumem um processo de transformação fundamental para o estabelecimento de sistemas sociotécnicos, que os compõem, buscando modos sustentáveis de produção e consumo (FARLA et al., 2012; MARKARD; RAVEN; TRUFFER, 2012). Em meio à discussão, emerge o consumo sustentável para a construção de novas relações sociais, compreendido como o padrão resultante de um conjunto de interações entre diferentes atores sociais (MICHAELIS, 2003; SILVA, 2012). Ao lidar com uma relação produção-consumo a temática é bem complexa e envolve aspectos abstratos como bem-estar, necessidades e futuras gerações em relação à sustentabilidade (CRIVITS et al., 2010; JACKSON, 2007).

Assim, para o desenvolvimento da presente discussão, o estudo parte da premissa de que em meio ao contexto de insustentáveis práticas coletivas sobre o meio, a incorporação de ações e estratégias de mudança pode contribuir para um processo de 'transição' macro. Desse modo, o objetivo deste artigo é compreender como a transição para a sustentabilidade, em meio a um contexto de crise, pode auxiliar para a promoção do consumo sustentável. Isso é possível ao se entender que para que um novo padrão de produção-consumo possa emergir efetivamente, algumas mudanças mais estruturais e disseminadas coletivamente devem ser incorporadas no sentido de haver construção de novas configurações sociais que levem a sociedade como um todo ao entendimento da sua relação direta com a situação atual.

Para tornar mais clara a relevância existente das temáticas estudadas, dois argumentos básicos devem ser considerados: (1) de acordo com Markard, Raven e Truffer (2012), hoje o campo de estudos sobre transição para a sustentabilidade tem recebido destaque nas agendas da comunidade acadêmica internacional, com isso entende-se que discussões no contexto brasileiro precisam ser realizadas, uma vez que são raros os estudos no país; e (2) o estudo do consumo sustentável apresenta grande avançado nas pesquisas desenvolvidas por toda a Europa, mas como salientam Schrader e Thøgersen (2011), os países emergentes podem trabalhar com o consumo sustentável na realização de seus estudos. Assim, entende-se que há vasta possibilidade de inserção do Brasil nas discussões sobre temas emergentes.

Quanto aos aspectos metodológicos, o estudo caracteriza-se como ensaio teórico, pois não se restringe a analisar o que a literatura versa sobre o assunto, mas configura-se como uma escrita reflexiva que busca estabelecer relações, convergir pensamentos e propor questionamentos que enriquecem o debate do assunto (MENEGHETTI, 2011). Corroborando, Severino (2007) afirma que o ensaio teórico é um processo discursivo formal que expõe o tema de maneira lógica e reflexiva, e 
complementa que dessa forma há a possibilidade de inserir interpretações pessoais. Considerando que as temáticas selecionadas para a construção deste estudo possui considerável amplitude, entende-se que a utilização dessa abordagem busca contribuir com uma visão mais clara e efetiva sobre tais discussões.

Diante dessas considerações, como forma de melhor compreender a relação teórica proposta, o artigo possui além dessa apresentação introdutória as seguintes seções: uma discussão sobre a sustentabilidade numa perspectiva de mudança destacando a compreensão dos princípios de transição sócio-técnica; a apresentação das insuficiências e potencialidades da abordagem do consumo sustentável; e a aproximação teórica entre a abordagem do consumo sustentável e a transição para a sustentabilidade, considerando-as complementares e articuladas. Além disso, como forma de reflexão sobre essas discussões, são apresentadas as considerações finais destacando as contribuições do artigo ao ter como relevante e importante o entendimento de convergências entre as temáticas.

\section{A Sustentabilidade e o contexto de Transição}

Os estudos envolvendo a sustentabilidade, cada vez mais estão sendo intensificados, ganhando destaque nas agendas de discussão, uma vez que leva em consideração a busca pela reestruturação dos impactos da atividade humana sobre a capacidade de carga do Planeta. Com sua ideia básica relacionada com uma mudança na atuação de diferentes atores voltados para a busca por uma harmonização entre os aspectos sociais, econômicos e ambientais, o desenvolvimento sustentável deve considerar a preocupação em atender as necessidades das gerações atuais e futuras (WCDE, 1987). Todavia, apesar dessas considerações, Elkington (2001) indica que o capitalismo e a sustentabilidade não compõem uma fácil aliança, o que sugere a necessidade de novos elementos para essa alternativa emergente.

Segundo Prothero e Fitchett (2000, p.48), "qualquer definição que usa entendimentos contemporâneos da natureza e das necessidades humanas para definir a sociedade verde não pode ser considerada distinta do modo de produção capitalista, uma vez que esses termos emergiram como parte das condições culturais do capitalismo". No entanto, deve-se entender que o argumento correto para o desenvolvimento sustentável não é a construção de um novo perfil ao declínio capitalista (SMITH, 2007), mas a busca pelo emergir de uma nova visão a partir da produção de consciência coletiva. Deve-se repensar o chamado mercado de carbono, por exemplo, o qual cria um novo mercado dando valor a um recurso natural. Essa perspectiva necessita de uma base para sua construção, a qual depende da forma como esta é observada.

Para Sachs (2008), a mudança no modelo de desenvolvimento consegue designar ao mesmo tempo o surgimento de subsídios para a sobrevivência humana no meio, bem como um novo enfoque de planejamento e gestão, no qual as práticas atuais redirecionam suas ações para questões mais amplas e coletivas, demonstrando um diferente papel a ser praticado pelos atores envolvidos. Isso se alinha com o que é apresentado por Hopwood, Mellor e O'brien (2005), no momento que os autores indicam diferentes abordagens para o desenvolvimento sustentável, a partir de um sentido transitório, no qual não se deve manter o status quo, como muitos pregam (o caso do 'esverdeamento' do capitalismo), mas deve-se buscar uma maior transformação, com a utilização de novas lentes e nova consciência.

Essa transformação pode assumir níveis de profundidade e impactar diretamente em como se dá o envolvimento de diversos atores em busca de sua implantação. É com relação essa noção que se trabalha com a abordagem teórica da transição para a sustentabilidade. Os estudos realizados sob essa vertente têm assumido bastante ênfase principalmente nos países europeus, no entanto como indicam Markard, Raven e Truffer (2012), está havendo um aumento na expansão geográfica de estudos sobre transição, o que certamente facilita que haja crescimento neste campo teórico. Para 
Geels (2011, p.37), "as transições podem ser estudadas para a análise de como práticas vem sendo desenvolvidas, como elas estão se estabilizando e como as práticas estabelecidas desaparecem".

Para entender o contexto que envolve a transição para a sustentabilidade, é necessário compreender qual a perspectiva que se tem sobre 'transição'. Como se observa na literatura a evolução sobre a temática considerou dentre outros aspectos o direcionamento da transição de unicamente tecnológica para uma visão sócio-técnica. Segundo Geels (2002), essa evolução considera que as transições tecnológicas (TT) - que eram entendidas como a transformação de caminhos na sociedade em diferentes sistemas - ao incorporar outros aspectos ao seu contexto criou uma perspectiva mais ampla e estrutural, entendida como transição sócio-técnica. A construção desse contexto estrutural leva a considerar que tudo o que é modificado envolve um conjunto de elementos efetivos, postos em prática (GEELS, 2010; 2011).

Geels (2002) indica que a TT em si consiste em uma mudança a partir de uma configuração sócio-técnica para outra, em relação à tecnologia e outros elementos. Para o autor esse processo de reconfiguração não ocorre facilmente, pela necessidade de relacionar os diferentes elementos existentes alinhados com outros. Para Markard, Raven e Truffer (2012, p.956), "as transições sociotécnicas diferem da transição tecnológica, pois incluem mudanças nas práticas dos usuários e na estrutura institucional (regulatória e cultural), para além da dimensão tecnológica". Entende-se então que existe uma complementaridade entre as transições, que a depender da profundidade que seja assumida, diferencia o impacto de seu movimento de mudança sobre a sociedade.

De acordo com o que sugerem Markard, Raven e Truffer (2012), a transição envolve uma gama de mudanças em diferentes dimensões: tecnológica, organizacional, institucional, material, política, econômica e sociocultural, os quais criam as configurações necessárias para a construção dos contextos que podem ser modificados. Nesse sentido, como apresenta Smith, Voв e Grin (2010), configurações sócio-técnicas em regimes específicos de mudança são estabelecidos como a forma estável e dominante de realizar determinada função social. Essa noção facilita o entendimento das transições para a sustentabilidade dentro de uma lógica de mudanças sistêmicas a partir de um processo contínuo (GEELS, 2011). Segundo o autor, tais modificações estão alinhadas com regimes de transformação.

Os regimes até então considerados, fazem parte de uma perspectiva de atuação multi atores que se envolvem na chamada perspectiva multi nível (PMN). Dividida em três níveis, a PMN considera a realização de mudanças de acordo com dinâmicas na sociedade, quais sejam: micro (nichos - onde surgem novas configurações); meso (onde mudam os regimes sociotécnicos - como regras); e macro (onde ocorre um envolvimento sociotécnico amplo - definidos como 'paisagens') (GEELS, 2002; 2010; SMITH, VOß; GRIN, 2010). Para tanto, são necessárias interações entre diferentes atores em busca de um melhor contexto de mudança na sociedade, os quais são apresentados na Figura 1, a partir de uma perspectiva complexa de interação entre os atores (FARLA et al., 2012; GEELS, 2002; 2011). 


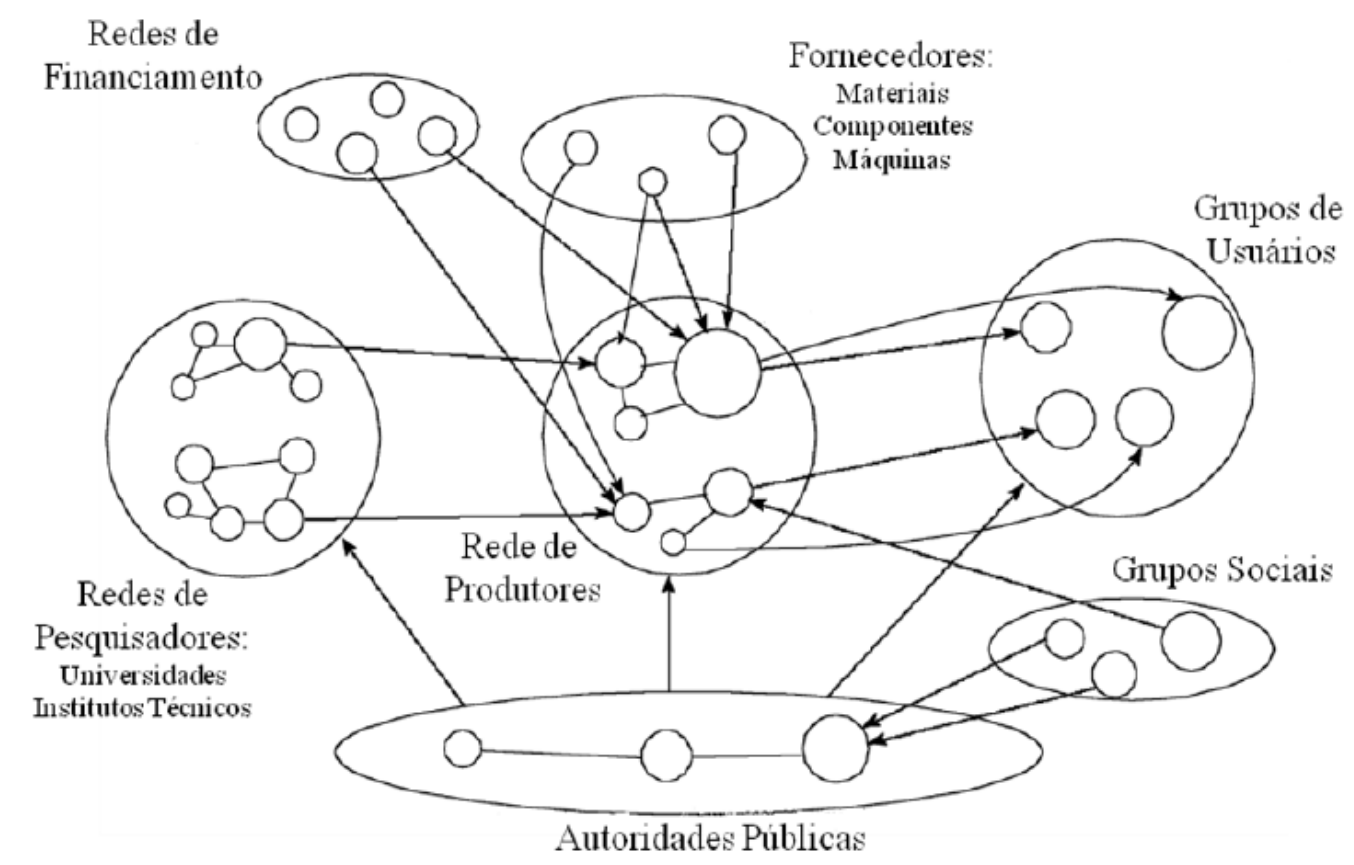

Figura 1: Rede multi atores envolvendo regimes sociotécnicos Fonte: Traduzido de Geels (2002, p.1260).

Como é apresentado na Figura 1, para a transição para a sustentabilidade - com a construção de configurações dentro dos regimes possíveis na PMN - deve-se considerar dentre os atores de impacto, grupos ou redes que levem à articulação dessas configurações. Para tanto, Geels (2011) traz as interações entre tecnologia, economia/negócio/mercado, política/poder, e cultura/discurso/opinião pública como básicas para que a perspectiva possa ser efetivada. Apesar dessa noção Smith, Voo e Grin (2010) e Geels (2011) entendem que existem críticas para a abordagem em vários sentidos: o papel da agência para as transições; a dificuldade de operacionalização; relações de poder nos regimes (principalmente nos nichos); dificuldades de definições metodológicas e epistemológicas. No entanto, existe uma noção clara sobre as ontologias que podem ser utilizadas (GEELS, 2010).

De acordo com Geels (2010), podem ser utilizadas sete ontologias: Escolha Racional; Teoria Evolucionária; Estruturalismo; Interpretativismo/Construtivismo; Funcionalismo; 'Conflitos e lutas de poder'; e Relacionismo. Assim, para questões deste estudo, assume-se a ontologia estruturalista, uma vez que considera a necessidade de lidar com mudanças institucionais e considera que os atores que atuam nesse contexto são observados como parte de coletivos sociais que compartilham um sistema de crenças, um conjunto de símbolos e categorias culturais que dão significados e senso de direção para a mudança (GEELS, 2010). Com isso, considera-se a necessidade de entendimento do processo de institucionalização.

Essa perspectiva considera uma mudança interna que leva a considerar o que se busca de fato no meio e não apenas o que ele exige das configurações. Com isso, dentro do contexto de transição podem ser trabalhados como vertentes de mudança: modernização ecológica, risco social, papéis governamentais, responsabilidade social corporativa, participação pública e democratização (GEELS, 2010). A partir de todas essas considerações, entende-se que numa perspectiva multi nível deve-se considerar os impactos gerados a partir dos multi atores e direcionar as ações da sociedade no sentido de alcançar modos de produção e consumo para a sustentabilidade. Para tanto, é necessário compreender a discussão sobre consumo sustentável, o qual necessita de um movimento transitório para ser efetivado. 


\section{Consumo Sustentável: Uma busca coletiva}

A partir da noção de atendimento das necessidades e desejos individuais, vive-se numa sociedade do consumo, no qual a busca pela felicidade é o aspecto principal pelo qual se consome (BAUDRILLARD, 2007). No entanto, segundo Lipovetsky (2007), percebe-se a existência de uma felicidade paradoxal, na medida em que o homo consumericus de uma sociedade emergente do hiperconsumo não considera as consequências de suas práticas insustentáveis. Surge desse modo o consumo exacerbado, consumismo, que é observado na maioria das populações ao redor do mundo, como parte da cultura de muitos países (ASSADOURIAN, 2010), e que indica a necessidade de uma mudança nos estilos de vida da população, para que seja possível a continuidade do consumo em escala mundial.

Corroborando essa ideia, Oliveira e Cândido (2010) indicam que atualmente essa prática pode ser considerada a responsável direta pelo desgaste ao sistema ambiental, já que desconsidera o aspecto finito e ameaçador imposto pelo sistema de produção atual. Assim, deve haver uma mudança nos comportamentos dos consumidores, o que para Michaelis (2000) envolve mais do que a simples redução do consumo, mas sim modificação em campos que influenciam no consumo, a saber: (1) tendências humanas intrínsecas - consumismo; (2) crescimento tecnológico e institucional; (3) consumismo material; (4) sistema de mercado competitivo; e (5) busca por qualidade de vida. 0 que indica a existência de outras ações, além do consumo, que devem ser consideradas nesse processo de reestruturação.

Nesse sentido, entende-se que no contexto social, a mudança nas práticas de consumo "reflete o momento cultural a qual a mesma [sociedade] está inserida, perante as dinâmicas e demandas requeridas, necessitando, assim, de um direcionamento coletivo para a efetivação de um desenvolvimento emergente, o sustentável" (SILVA, 2011, p.29). Assim, segundo a CNUMAD (1992) é necessário que haja a promoção de padrões de consumo e produção que reduzam as pressões ambientais e atendam às necessidade da humanidade, as quais devem focar o contexto de países, o que é corroborado por Tukker et al. (2008) quando os autores indicam a necessidade de construção de novos padrões de consumo em diferentes níveis de economia desde as desenvolvidas e em desenvolvimento até de base da pirâmide.

O marco das discussões sobre a necessidade de um novo padrão de consumo foi o documento Agenda 21 resultante da Conferência Eco-92, no qual se destaca a dificuldade de atendimento das necessidades básicas humanas, o que ainda pode ser visualizado depois da Conferência Rio+20. Para tanto, a "mudança dos padrões de consumo exigirá uma estratégia multifacetada centrada na demanda, no atendimento das necessidades básicas dos pobres e na redução do desperdício e do uso de recursos finitos no processo de produção" (CNUMAD, 1992, n.p.). É nesse contexto que se embasa a criação do conceito de consumo sustentável, que emerge das demandas de consumidores, mas necessita de reestruturação em todas as relações de consumo que permeiam o atendimento dessas necessidades.

No entanto, ainda existem confusões quanto ao consumo voltado à sustentabilidade, as quais muitas vezes se sobrepõem. Como destaca Silva (2012), os tipos de consumo não são iguais e seguem uma sequência hierárquica que parte do consumo verde ao considerar especificamente o impacto ambiental do produto/serviço consumido (GILG; BARR; FORD, 2005; PORTILHO, 2005), passa pelo consumo consciente que está relacionado a reflexão individual sobre seu impacto na sociedade (SILVA; MENK, 2012), até chegar ao consumo sustentável que deve ser considerado como uma abordagem macro, mais estrutural que considera a mudança nas ações, práticas e comportamentos de outros atores e contextos (GLAVIC; LUKMAN, 2007). Todos devendo considerar a relação entre produção e consumo existente, o que para Mont (2003) deve passar por um processo de institucionalização.

Assim, frente a esse contexto, cada ator necessita assumir responsabilidades distintas, mas convergentes ao objetivo maior que envolve a reestruturação nos padrões de consumo utilizados. 
Nessa perspectiva, além das empresas que estão inseridas em diferentes estruturas e que possuem diferentes comportamentos no mercado, outros atores podem ser visualizados nesse sistema, direcionando-se para o consumo sustentável, dentre os quais se identificam: os fornecedores, os competidores, organizações do terceiro setor, as instituições financiadoras, as universidades, a mídia, o governo e os indivíduos (dentro do contexto da comunidade) (MICHAELIS, 2003; MONT; PLEPYS, 2007; SILVA, 2011). Os quais podem ser melhor observados na Figura 2 a seguir:

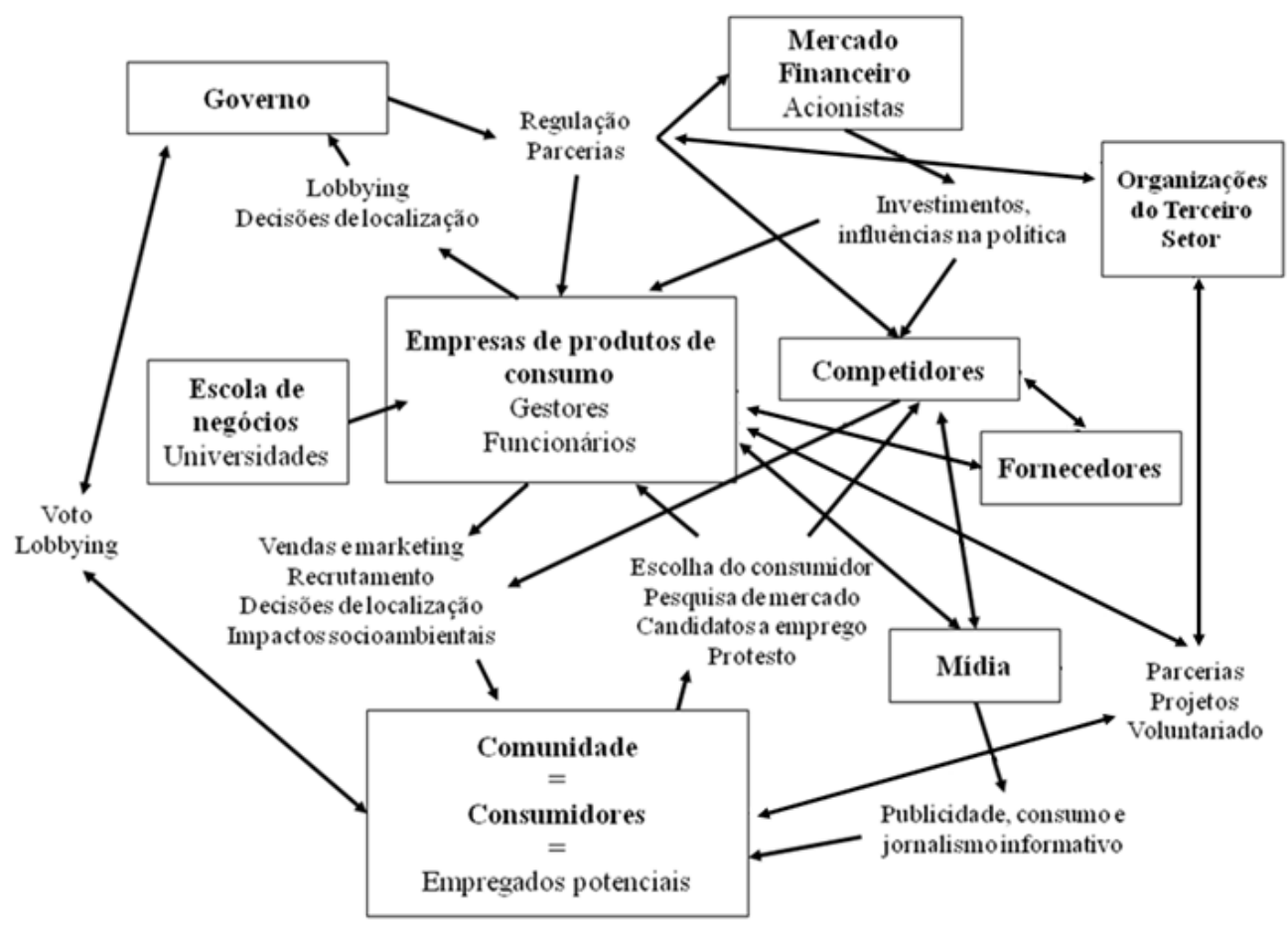

Figura 2: Mapa da rede de influências para o consumo sustentável Fonte: Silva (2011)

Apesar dessa visão e da definição de algumas ações para cada ator social, pouco de efetivo ainda se tem sobre posicionamento e responsabilidades assumidos individualmente. A abordagem emergente do consumo sustentável ainda apresenta-se como ineficiente em sua efetivação, já que ainda não possui plena consistência teórica para seu estudo. Existe a busca por essa consistência em diversos sentidos, inicialmente no que se refere ao embasamento empírico necessário para ser observado, o que ratifica mais a possibilidade de contribuição desse ensaio para os estudos acadêmicos. Esse fato, para Silva (2012), pode ser minimizado a partir do aprofundamento teórico e de pesquisas que possam facilitar a compreensão de como atuam os diferentes atores sociais antes apresentados.

De acordo com Rossetti e Giacomini Filho (2010, p.168), não se "chegou a um consenso social sobre o consumo sustentável, mas este se aloja numa nova correlação de forças amparadas em avanços consensuais favoráveis às causas ambientais, que seria uma contínua terceira via que não despreza a racionalidade em suas ponderações", com o atual conceito, em construção, sendo originário de um processo consensual conduzido por organizações, pessoas e sistemas de comunicação. Mesmo assim, segundo Silva (2012), o consumo sustentável pode ser compreendido e conceituado como o padrão de consumo resultante de inter-relações sociais sob a perspectiva de interação política entre diferentes atores sociais direcionados ao alcance do desenvolvimento sustentável. 
Para o autor, isso se torna possível por meio da coordenação de práticas e relações existentes na dinâmica do consumo sustentável. Em geral, a comunidade empresarial tem interpretado o consumo sustentável como apenas o consumo de produtos sustentáveis ou ecologicamente corretos, todavia o que se percebe é que existe uma complexidade maior que circunda a prática sustentável do consumo, por esse motivo é que se discute a ideia de interações sociais (MICHAELIS, 2003). Corroborando, Málovics, Csigéné e Kraus (2008) afirmam a possibilidade de atuação das empresas na direção de um consumo mais ou menos sustentável, devendo-se observar os comportamentos adotados pelas mesmas.

Essa ideia leva a compreensão de que quando uma prática é incorporada por uma empresa, por exemplo, essas, por meio das ações sociais, impulsionam mudanças naqueles que estão participando do seu campo. Desse modo, busca-se por meio da institucionalização: a minimização das incertezas, a busca por certa estabilidade e a ratificação de que esse ator que se utilizou da característica institucional consegue estar atuando em novas relações, no caso para a prática do consumo (DIMAGGIO; POWELL, 2005). Tais aspectos dão a visão sobre quais as potencialidades que a abordagem possui para ser efetivada, isso porque facilita a possibilidade de operacionalização da sustentabilidade, ao se relacionar diretamente com o mesmo e pressupor diferentes relações modificadas no mercado.

\section{O movimento de transição para o consumo sustentável}

A partir do entendimento teórico até então apresentado, fica evidente que em busca de uma melhor atuação da sociedade na redução de seus impactos sobre o meio, muitas ações devem ser tomadas e estratégias elaboradas. Isso porque está na organização e no processo de gestão o caminho mais propenso ao alcance de mudanças amplas. Para o consumo sustentável ser efetivado em diferentes localidades, uma reestruturação de comportamentos multi atores deve ser realizada, uma vez que esse tema incorpora e abrange não apenas o consumo em si, mas considera também a influência do processo de produção e distribuição em toda a sua compreensão. Com isso, percebe-se a total e necessária contribuição do processo de transição, à medida que o mesmo deve ser entendido como contínuo e sistêmico (GEELS, 2011).

De acordo com o CNUMAD (1992, n.p.), para promover ações mais positivas em relação ao consumo sustentável, os governos e organizações privadas, enquanto os atores com maior destaque, devem direcionar suas ações para a "educação, programas de esclarecimento do público e outros meios, como publicidade positiva de produtos e serviços que utilizem tecnologias ambientalmente saudáveis ou estímulo a padrões sustentáveis de produção e consumo". Isso se torna possível a partir da realização de práticas responsáveis, que podem ser facilitadas a partir do processo de institucionalização, de acordo com um posicionamento voltado para o contexto emergente.

Essa ideia pode receber contribuições de ações realizadas por outros diferentes atores, o que exige a consideração de um reposicionamento de cada um desses atores para ser efetivo o processo de reconfiguração. É nesse momento que devem ser considerados os aspectos de mudança apresentados pela abordagem de transição. Apesar dessa noção, Geels (2010) indica que as transições que podem contribuir para um contexto de sustentabilidade não ocorrem facilmente, uma vez que envolvem sistemas de alto impacto na sociedade. Segundo o autor, dentre os sistemas que estão sendo estudados e servem de referência para tal afirmação estão a distribuição de água e energia, o transporte e a alimentação. De fato são sistemas complexos que necessitam de uma transformação articulada, de acordo com as soluções que são criadas.

Como mencionado na discussão anterior sobre transição, sob uma perspectiva multi nível ações devem ser realizadas por diferentes atores em busca de novas configurações sócio-técnicas que impactam de diferentes modos na sociedade. Toda a discussão sobre transição baseia-se nas inovações, sejam essas radicais ou não, que contribuem com novos contextos e podem redefinir os regimes utilizados. Se o foco das mudanças estiver na construção de um novo nicho para uma 
inovação radical, fica claro que se houver uma tecnologia efetiva na busca por novas soluções, é possível que haja uma transição que saia de um campo micro de mudança, passe ao meso e possa chegar ao campo macro, com a criação de novas 'paisagens' que levam a construção de regimes sócio-técnicos prolongados.

A cada nível de transição (micro-meso-macro) as configurações sócio-técnicas levam a considerar quais seriam os regimes reais em determinado contexto de transição. Em meio a essa discussão, Geels (2002) ressalta que a mudança (com a inserção de inovações) pode ocorrer nas relações e ser entendida pela sociedade. Nesse caso, as regras de mudança podem estar mais desenvolvidas do que o contexto macro - a 'paisagem' criada. Seguindo a PMN pode haver uma perspectiva hierárquica, na qual se busca de fato uma visão de continuidade, no entanto muitas vezes mudanças na perspectiva estável levam apenas a um campo onde regras podem mudar e não necessariamente o contexto exógeno. Com isso, observar o fluxo contínuo de mudanças (Figura 3), demonstra como tudo pode ser considerado.

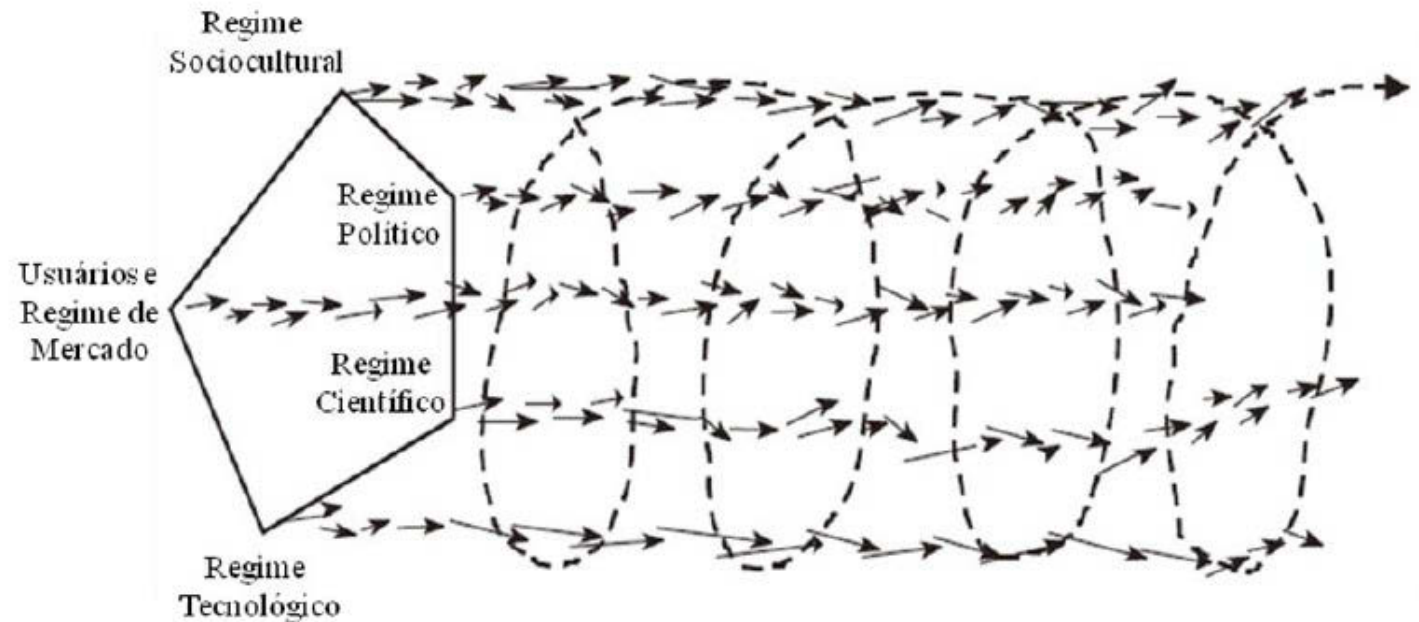

Figura 3: Alinhamento do processo contínuo no regime sociotécnico Fonte: Traduzido de Geels (2011, p.27).

Como se observa na ilustração, cada uma das vertentes do pentágono representa um tipo de regime que pode estar influenciando na efetivação de uma transição. Esses regimes são desenvolvidos continuamente e de forma sistêmica, assim sendo, a depender de como são considerados os níveis da PMN em relação às configurações que são criadas e ao perfil sócio-técnico delineado como base para a mudança, torna-se efetiva a base de uma transição para a sustentabilidade. Ou seja, a construção de uma 'paisagem' nova na sociedade. Para Smith, Voв e Grin (2010), tal perspectiva contribui para um contexto estrutural de regimes e nichos, criando a relação direta entre os níveis. Segundos os autores, essa visão macro cria funções sociais e autonomia para determinados regimes sócio-técnicos.

Segundo Geels (2002, p, 1259), "mudanças em um elemento na rede pode desencadear mudanças em outros elementos". Essa ideia está alinhada com o que foi discutido até então sobre a necessidade de um movimento de interação hierárquico e multi nível focado no processo de mudança. Como Geels (2011) indica, dependendo do tema de pesquisa, se for em micro-eventos podem ocorrer movimentos e conta-movimentos de atores, mas se for em macro-eventos (como a Revolução Industrial) consideram-se várias mudanças. Para o autor esse evento ocorreu por uma sequência de três eventos: uma ambiental, uma cultural e uma de industrialização. Assim, em meio a noção do consumo sustentável, à medida que novas responsabilidade individualmente foram 
assumidas e realizadas essas podem contribuir para uma mudança coletiva, na construção de um padrão coletivo.

Discute-se que desde a Revolução Industrial, só puderam ser postos em prática e desenvolvida as novas formas de produção e tecnologias, na medida em que houve uma revolução do consumo concomitante a essa mudança nos meios de produção (CAMPBELL, 2001). Para o autor, a partir de uma mudança nos valores e nas atitudes dos consumidores, ou seja, de seu contexto cultural é que começaram a se desenvolver a revolução do consumo e, por conseguinte, as evoluções ditas inerentes a Revolução Industrial. Se houve essa mudança cultural que impulsionou uma reestruturação social para uma nova forma de produzir e consumir, por que não o fazê-lo novamente direcionado para as questões do desenvolvimento sustentável? A perspectiva do consumo sustentável discute exatamente esse ponto.

Markard, Raven e Truffer (2012) relembram que para haver uma 'transição' se faz necessário um processo de institucionalização, principalmente no contexto estruturalista. Assim, para a aproximação entre as vertentes aqui consideradas, assume-se o mapeamento de possíveis atores que podem atuar no processo de construção de relações sociais apresentados na Figura 2, como o conjunto de interações que representam o campo organizacional de uma determinada organização empresarial. Isso porque, para DiMaggio e Powell (2005), o campo organizacional surge como resultado de atividades de um grupo diverso de organizações, que seguido de um processo de homogeneização. Segundo os autores, esse campo não deveria ser definido a priori, no entanto como tal mapeamento é resultado de pesquisas empíricas pode-se considerá-lo como válido.

Existem várias possibilidades de práticas que podem ser institucionalizadas, como já citado. Dentro do contexto de transição podem ser trabalhados como vertentes de mudança: modernização ecológica, papéis governamentais, responsabilidade social corporativa (RSC), risco social, participação pública e democratização (GEELS, 2010). A atuação de multi atores pode ser observada, mas para o desenvolvimento da presente aproximação, discute-se o papel das empresas a partir da RSC. Deve-se entender que o processo de institucionalizar práticas para uma responsabilidade social não considera apenas a influência da empresa no todo, mas existe a necessidade de uma mudança em vários sentidos com a construção de configurações sócio-técnicas que levem a uma melhor dinâmica daquele conjunto de atores.

Schultz e Wehmeir (2010) indicam que a institucionalização de práticas responsáveis perpassa três fases, ou seja, níveis de institucionalização da responsabilidade empresarial, a qual se observada sobre o contexto de interação entre os atores e capacidade de agência empresarial, facilitam a adequação e contribuição da empresa para o consumo sustentável. Segundo os autores, podem ser analisados os níveis: Micro (individual) que foca o indivíduo dentro da organização; Meso (organizacional) que foca as práticas organizacionais, o que envolve desde os valores, cultura, até interpretação interna; e, por fim, Macro (ambiental) que foca as interações organizacionais indicando a possibilidade de imitação entre as mesmas.

Segundo Silva (2011) para que se possa contribuir com o consumo sustentável, em meio ao conjunto de interações sociais, é necessário que haja a incorporação de práticas responsáveis, as quais devem permear dentre outras atividades: Eco-eficiência, Práticas próprias de consumo, Incentivos do governo, Elos da cadeia de suprimentos, Edição de escolha, Cidadania corporativa, Marketing responsável e Diálogo com stakeholders. Tais práticas se forem institucionalizadas, direcionam a atuação empresarial para um contexto de estabilidade em relação ao seu campo organizacional no que se refere a mudança, a contribuição para a construção de novas relações de consumo, e como indica Mont (2003) na incorporação de estratégias de produção e consumo mais responsáveis.

Assim percebe-se um alinhamento com o que a abordagem de transição traz sobre a necessidade de movimentos que sofrem influências e influenciam as relações em determinado contexto. Para Crivits et al. (2010), devem ser criados cenários no sentido de buscar sistemas direcionados ao consumo sustentável. Como já mencionado, esses sistemas são complexos e 
necessitam de diferentes estratégias de atuação. Farla et al. (2012) trazem alguns sistemas que estão sendo estudados numa perspectiva de longo prazo, dentre os quais pode-se observar o setor automobilístico, cuidados de saúde, bem como o abastecimento de energia, destacado também por Geels (2002). Sobre esse último sistema existe trabalhos brasileiros que estão analisando-o. Assim, ao observar a Figura 4, consegue-se entender o direcionamento para o consumo sustentável no setor e tentar fazer uma relação direta com a ideia de 'transição'.

\begin{tabular}{|c|c|}
\hline Stakeholder identificado & Contribuição para o consumo sustentável \\
\hline Governo & $\begin{array}{l}\text { Seu papel foi implantar nova lei focando o estímulo a práticas de consumo de } \\
\text { energia mais responsáveis. Além disso, ele pode ser relacionado com a criação } \\
\text { de políticas públicas tanto para a população quanto para o mercado. }\end{array}$ \\
\hline $\begin{array}{c}\text { CELPE (Companhia } \\
\text { estudada) }\end{array}$ & $\begin{array}{l}\text { Seu papel foi se adequar a essa lei e incorporar novas práticas em suas rotinas } \\
\text { visando novas relações de consumo. Além disso, a empresa criou parcerias } \\
\text { alinhadas com suas metas, visando o alcance efetivo do consumo sustentável. }\end{array}$ \\
\hline ONGs & $\begin{array}{l}\text { Estiveram envolvidos com o processo de sensibilização e encontros para os } \\
\text { objetivos dos projetos, estimulando uma nova prática de consumo, a partir das } \\
\text { parcerias efetivas. }\end{array}$ \\
\hline Mídia & $\begin{array}{l}\text { São responsáveis pela disseminação das práticas da companhia, bem como } \\
\text { estimular novas práticas de consumo enfatizando a ideia de consumo de } \\
\text { energia. Isso ocorre na mídia em massa (impressa ou online) ou em } \\
\text { comentários privativos na internet. }\end{array}$ \\
\hline Consumidores & $\begin{array}{l}\text { Seu papel está relacionado com a disponibilidade e efetividade de mudança } \\
\text { em seu comportamento de consumo, uma vez que dá continuidade de seu } \\
\text { consumo ao longo do tempo. Adicionalmente, teve o papel de legitimar as } \\
\text { práticas da empresa e envolver voluntários no processo de sensibilização. } \\
\text { Além disso, deve realizar pressão sobre empresas por seu comportamento, } \\
\text { expandindo essas práticas conscientes de consumo para outros setores. }\end{array}$ \\
\hline $\begin{array}{l}\text { Banco do Brasil } \\
\text { (representante do mercado } \\
\text { financeiro) }\end{array}$ & $\begin{array}{l}\text { Inicialmente teve o papel de facilitar o financiamento de refrigeradores para as } \\
\text { familias, contribuindo para que a companhia pudesse atender seus objetivos } \\
\text { delineados em seu projeto. }\end{array}$ \\
\hline Funcionários & $\begin{array}{l}\text { Por meio do projeto Agente Celpe, os funcionários disseminaram o projeto } \\
\text { por todo o Estado, identificando potenciais beneficiários e facilitando a } \\
\text { interação da companhia com os consumidores de energia. }\end{array}$ \\
\hline
\end{tabular}

Figura 4: Papéis identificados para o consumo sustentável

Fonte: Traduzido de Silva, Oliveira e Gómez (2013).

Para chegar numa compreensão do sistema de distribuição energético brasileiro, Silva, Oliveira e Gómez (2013) realizam um levantamento dos fatores que contribuíram para uma mudança de ação na rede de atores setorial. O caso estudado pode ser considerado como um micro-evento que observa movimentos e contra-movimentos de multi atores - ao estudar apenas o Projeto Nova Geladeira, no entanto apresenta o caminho seguido para a realização das mudanças. Existiu um grande período para que houvesse uma construção e um direcionamento de todos esses atores voltados para o consumo sustentável no setor de energia. Esse período partiu de uma mudança institucional com a criação de um conjunto de novas leis que contribuíram para a dinamicidade do setor nos últimos anos (SILVA; GÓMEZ, 2011).

Como apresentado por Silva, Oliveira e Gómez (2013), não é possível considerar a existência de consumo sustentável, considerando a necessidade de construção de padrão de consumo inerente ao entendimento teórico desta abordagem. No entanto, entende-se que a partir de um processo de transição com a busca por novas configurações sócio-técnicas nas interações sociais observadas, existe a possibilidade de alcance desta sustentabilidade. Como indica a lei de responsabilidade social no setor, devem haver investimentos em diversas áreas sejam elas Pesquisa \& Desenvolvimento ou 
criação de inovações completas. Entende-se que o processo de mudança na perspectiva da estrutura que envolve o setor, facilita que a transição possa ser almejada e que discussões mais profundas e futuras possam ser realizadas.

Apesar disso, baseando-se nas informações da Figura, identifica-se a construção de uma mudança no comportamento dos atores, o que pode estar relacionado com a construção de configurações sócio-técnicas que levaram às reestruturações no setor. Ou seja, práticas e ações responsáveis estão sendo institucionalizadas e tem levado ao entendimento de que na busca por uma continuidade das atividades do setor, bem como no sentido de busca por um consumo sustentável essa movimentação de interações multi atores, pode levar a promoção desse novo padrão de consumo e uma reconfiguração da 'paisagem'. A partir de todas as discussões realizadas, percebe-se que a transição para a sustentabilidade pode contribuir de forma direta para a efetivação de um consumo sustentável, considerando as ações criadas e as mudanças estimuladas por novos posicionamentos.

\section{Considerações Finais}

No entendimento de que existe possibilidade de convergência entre as abordagens de transição para a sustentabilidade e do consumo sustentável, o presente estudo buscou articular um pensamento sobre relações teóricas pouco realizadas. Entende-se que são abordagens que apresenta grande complexidade permeando as possibilidades de visualização empírica, mas como foi apresentado, o texto buscou apresentar uma dinâmica nova. Para Geels (2010) as transições para a sustentabilidade se apresentam como um tópico rico e desafiador, mas que são benéficas para a realização de diálogos entre as abordagens. Salienta-se que a discussão não teve a intenção de realizar um aprofundamento exaustivo das temáticas, mas demonstrar que as relações podem ser realizadas e melhor observadas no contexto social.

Fica claro ao longo do estudo sobre as temáticas que de forma ampla o mesmo pode ser trabalhado dentro da configuração de mudança, no entanto como indicam Markard, Raven e Truffer (2012) apesar dessa noção, refletindo as críticas existentes para a abordagem são necessárias mais pesquisas que empírica e conceitualmente consigam trazer novas relações para a 'transição'. Com isso, para os autores os estudos devem ser guiados por quatro campos básicos: gestão da transição, gestão de nichos estratégicos, perspectiva multi nível e sistemas de inovação tecnológica. O presente trabalho se encaixa no terceiro campo de estudo, à medida que busca contribuir para a construções de relações pouco observadas numa visão multi níveis e multi atores, como sugerido por Farla et al. (2012).

A partir dos aspectos supracitados, este ensaio teórico alcançou seu objetivo de estudo, pois ao longo da articulação entre as temáticas buscou compreender como a transição pode se apresentar como um movimento efetivo voltado para a efetivação do consumo sustentável. $\mathrm{Na}$ discussão, não foi dada muita ênfase a questão no momento de crise, uma vez que ao ter a sustentabilidade como base para toda a discussão considera-se que tal aspecto seja intrínseco. Assim, esta discussão apresenta uma contribuição para os estudos acadêmicos ao trazer a tona uma discussão pouco realizada no contexto brasileiro. São duas temáticas ainda com pouca ou total consistência teórica, mas que demonstram direções e estratégias de pesquisa.

Com essa noção, esta se apresenta como a maior limitação de pesquisa pela falta de disseminação nacional sobre as temáticas, o que à necessidade de consideração desta baixa consistência teórica. Novas discussões devem ser realizadas no sentido de melhorar a base de discussão sobre as temáticas. Além disso, devem ser realizadas pesquisas empíricas sobre o tema que considerem ao longo de sua realização aspectos relacionados à transição, bem como replicados estudos que levem em consideração os mesmos sistemas analisados em trabalhos publicados no setor automobilístico, na distribuição de água, além de intensificar estudos sobre a distribuição de energia no Brasil para entender se está havendo um processo de transição para a sustentabilidade. 


\section{Referências}

ASSADOURIAN, E. Ascensão e queda das culturas de consumo. In: WORLDWATCH INSTITUTE. Estado do Mundo, 2010: estado do consumo e o consumo sustentável. Salvador: Uma Ed., 2010.

BAUDRILLARD, J. A sociedade de consumo. Lisboa: Arte \& Comunicação, 2007.

CAMPBELL, C. A ética romântica e o espírito do consumidor moderno. Rio de Janeiro: Rocco. 2001.

CNUMAD - Conferência das Nações Unidas sobre Meio Ambiente e Desenvolvimento. Agenda 21 Global. 1992. Disponível em:

$<$ http://www.mma.gov.br/sitio/index.php?ido=conteudo.monta\&idEstrutura=18\&idConteudo=575\&i dMenu=9065>. Acesso em: Set. 2012.

CRIVITS, M.; PAREDIS, E.; BOULANGER, P-M.; MUTOMBO, E. J. K.; BAULER, T.; LEFIN, A-L. Scenarios based on sustainability discourses: constructing alternative consumption and consumer perspective, Futures, 42, p.1187-1199, 2010.

DIMAGGIO, P. J.; POWELL, W. W. A gaiola de ferro revisitada: isomorfismo institucional e racionalidade coletiva nos campos organizacionais, Revista de Administração de Empresas - RAE, 45 (2), p.74-89, 2005.

ELKINGTON, J. Canibais com garfo e faca. São Paulo: Makroon Books, 2001.

FARLA, J.; MARKARD, J.; RAVEN, R.; COEREN, L. Sustainability transitions in the making: a closer look at actors, strategies and resources, Technological Forecasting \& Social Change, 79, p.991-998, 2012.

FOLADORI, G. Por uma sustentabilidad alternativa. Uruguai: Colección Cabichui, 2005.

GEELS, F. W. Ontologies, socio-technical transitions (to sustainability), and the multi-level perspective, Research Policy, 39, p.495-510, 2010.

GEELS, F. W. Technological transitions as evolutionary reconfiguration processes: a multi-level perspective and a case-study, Research Policy, 31, p.1257-1274, 2002.

GEELS, F. W. The multi-level perspective on sustainability transitions: Responses to seven criticisms, Environmental Innovation and Societal Transitions, 1, p.24-40, 2011.

GILG, A.; BARR, S.; FORD, N. Green consumption or sustainable lifestyle? Identifying the sustainable consumption, Futures, 37, pp. 481-504. 2005.

GLAVIC, P.; LUKMAN, R. Review of sustainability terms and their definitions, Journal of Cleaner Production, 15, p. 1875-1885. 2007.

HOPWOOD, B.; MELLOR, M.; O'BRIEN, G. Sustainable Development: Mapping Different Approaches, Sustainable Development, 13, 2005.

JACKSON, T. Sustainable Consumption. In: ATKINSON, G.; DIETZ, S.; NEUMAYER, E. (editors). Handbook of Sustainable Development. Edward Elgar Publishing, 2007. 
LAWHON, M.; MURPHY, J. T. Social-technical regimes and sustainability transitions: insights from political ecology, Progress in Human Geography, 36 (3), 2011.

LIPOVETSKY, G. A felicidade paradoxal: ensaio sobre a sociedade de hiperconsumo. São Paulo: Companhia das Letras, 2007.

MÁLOVICS, G.; CSIGÉNÉ, N. N.; KRAUS, S. The role of corporate social responsibility in strong sustainability, The Journal of Socio-Economics, 37, p.907-918, 2008.

MARKARD, J.; RAVEN, R.; TRUFFER, B. Sustainability transitions: an emerging field of research and its prospects, Research Policy, 41, p.955-967, 2012.

MENEGHETTI, F. K. O que é um ensaio teórico? Revista de Administração Contemporânea - RAC. 15 (2), p.320-332. 2011.

MICHAELIS, L. Ethics of consumption. Ethics \& Society. 2000. Disponível em:

<http://spin.medic.ukm.my/Contents/ZZZT3133/ZZZT3133/CModule/Lesson13/1315344428_Ethicso fConsumption.pdf> Acesso em: Nov. 2011.

MICHAELIS, L. The role of business in sustainable consumption, Journal of Cleaner Production, 11, 2003.

MONT, O. Institutionalisation of sustainable consumption patterns based on shared use, Ecological Economics, 50, p. 135-153, 2003.

MONT, O.; PLEPYS, A. Sustainable consumption progress: should we be proud or alarmed? Journal Cleaner Production, V.16, p.531-537, 2007.

OLIVEIRA, V. M.; CÂNDIDO, G. A. Contemporaneidade do Consumo Sustentável e as suas correlações com as Práticas Empresariais e o Comportamento do Consumidor. Anais do V Encontro Nacional da ANPPAS. Florianópolis: 2010.

ONARAN, Ö. The crisis of capitalism in Europe, West and East. Monthly Review. 2010. Disponível em: http://monthlyreview.org/author/ozlemonaran. Acesso em: abr. 2012.

PORTILHO, M. F. F. Sustentabilidade Ambiental, consumo e cidadania. São Paulo: Cortez, 2005.

PROTHERO, A.; FITCHETT, J. A. Greening Capitalism: opportunities for a green commodity, Journal of Macromarketing, 20, 2000.

ROSSETTI, R.; GIACOMINI FILHO, G. Comunicação, consenso social e consumo sustentável, Comunicação, mídia e consumo, 7 (18), p. 153-169, 2010.

SACHS, I. Caminhos para o desenvolvimento sustentável. Rio de Janeiro: Garamond, 2008.

SCHRADER, U.; TH $\varnothing$ GERSEN, J. Putting sustainable consumption into practice, Journal of consumer policy, Springer Science, 2011.

SCHULTZ, F.; WEHMEIER, S. Institutionalisation of corporate social responsibility within corporate communications: Combining institutional, sensemaking and communication perspectives, Corporate communication: An International Journal, 15, p.9-29, 2010. 
SEVERINO, A. Metodologia do trabalho científico. 23. ed. São Paulo: Cortez, 2007.

SILVA, M. E. A contribuição de práticas empresariais responsáveis para o consumo sustentável no varejo de supermercados: 0 caso Walmart Brasil. 137p. Dissertação (Mestrado em Administração) Programa de Pós-Graduação em Administração (PROPAD). Universidade Federal de Pernambuco, Recife: 2011.

SILVA, M. E. Consumo Sustentável: A Articulação de um constructo sob a perspectiva do desenvolvimento sustentável, RECADM: Revista Eletrônica de Ciências Administrativas, 11 (2), 2012.

SILVA, M. E.; GÓMEZ, C. R. P. O papel do governo e a prática do consumo sustentável: Como esse stakeholder atua no setor elétrico? RECADM: Revista Eletrônica de Ciência Administrativa. 10 (2), 107-123. 2011.

SILVA, M. E.; MENK, T. F. A Prática do Consumo e os Níveis de Consciência: Um ensaio sobre Consumo Consciente sob a perspectiva filosófica de Hegel. Anais do VI Encontro Nacional de Estudos do Consumo e II Encontro Luso-Brasileiro de Estudos do Consumo. Rio de Janeiro, 2012.

SILVA, M. E.; OLIVEIRA, A. P. G.; GÓMEZ, C. R. P. Can collaboration between firms and stakeholders stimulate sustainable consumption? Discussing roles in the Brazilian electricity sector, Journal of Cleaner Production, In press, 2013. http://dx.doi.org/10.1016/j.jclepro.2012.10.027

SMITH, A.; VOß, J-P.; GRIN, J. Innovation studies and sustainability transitions: the allure of the multilevel perspective and its challenges, Research Policy, 39, p.435-448, 2010.

SMITH, N. Nature as accumulation strategy, Socialist Register, 43, 2007.

TUKKER, A.; EMMERT, S.; CHARTER, M.; VEZZOLI, C.; STO, E.; ANDERSEN, M. M.; GEERKEN, T.; TISCHNER, U.; LAHLOU, S. Fostering change of sustainable consumption and production: an evidence based view, Journal of cleaner production, 16, 2008.

VANDEPITTE, M. Crisis del capitalismo. 2011. Rebelión. Disponível em: http://www.rebelion.org/noticia.php?id=121086 Acesso em: mai. 2012.

WCED - World Commission on Environment and Development. Report Our common future. Genebra, 1987. Disponível em: <http://www.un-documents.net/wced-ocf.htm.>. 